\title{
Living cell-based gas sensor system for the detection of acetone in air
}

\author{
Ulrich Bohrn ${ }^{1}$, Evamaria Stütz ${ }^{1}$, Maximilian Fleischer ${ }^{1}$, Michael J. Schöning ${ }^{2}$, Patrick Wagner ${ }^{3}$ \\ ${ }^{1}$ Siemens AG, Corporate Research \& Technologies, Otto-Hahn-Ring 6, Munich, Germany, \\ ulrichbohrn@hotmail.com \\ ${ }^{2}$ Aachen University of Applied Sciences, Institute of Nano- and Biotechnologies (INB), Heinrich- \\ Mussmann-Straße 1, Jülich, Germany, \\ ${ }^{3}$ Hasselt University, Institute for Material Science (IMO/IMOMEC), Wetenschapspark 1, Diepenbeek, \\ Belgium
}

\begin{abstract}
:
In this study, a cell-based gas biosensor is presented, which can be used for the detection of gaseous organic compounds in ambient air. The response of living human nasal cells (RPMI 2650) towards the direct exposure of gaseous substances is monitored with a multi-parametric sensor system using an exposure time of 10-15 minutes. Changes in the cellular impedance, oxygen consumption rate and acidification rate are recorded immediately after the exposure and represent the metabolic cell reaction to the gas presented. The system is able to notify the presence of acetone in aqueous solution $(\sim 2 \%)$ but also - in significantly lower concentrations - in the gas phase ( 200 ppm) within 30 minutes. The concentration-dependent decrease of cellular impedance is caused by the lipophilicity of this solvent, which causes hydrophobic interactions within the protein structure of the cell. The system might be used in the future for the monitoring of ambient air in work spaces as well as for the characterization of medical gases and aerosols.
\end{abstract}

Key words: cell-based gas biosensor, acetone, impedance, respiration, acidification

\section{Introduction}

Sensor systems based on living cells are primarily used in the detection of bioactive substances in aquatic environments, like waste water monitoring [1] or screening of potential drugs that are solved in liquid nutrient medium [2]. Latest studies demonstrated the feasibility of cell-based sensors for the detection of toxic gases (e.g., $\mathrm{CO}$ and $\mathrm{NH}_{3}$ ) in air [3,4]. These types of sensors are non-specific in the sense that they can respond towards a large range of toxic substances and are therefore intended to determine integral parameters, like the 'toxicity' of a sample. Their universal applicability makes them ideal sensors for the detection of unexpected toxic compounds in the gaseous environment.

Volatile organic compounds (VOCs) are a class of gaseous pollutants which are widespread in ambient air. Sources for VOCs are cleaning processes, carpets and furniture, cigarette smoke, etc. Acetone is a colorless, highly volatile and flammable liquid that is used in numerous industrial processes as well as an intermediate for organic molecules, like bisphenol $A$ and as a solvent. Acetone is not considered to be toxic itself as it is produced endogenously by the human body. However, accidental exposure to acetone concentrations larger than $10,000 \mathrm{ppm}$ is reported to cause severe sensory irritations, like burning sensation or rapid breathing [5]. Due to its lipophilicity, acetone can readily cross the cellular membrane. The aim of the study was to characterize a cell-based gas sensor system regarding its ability to detect non-acute-toxic, volatile organic compounds.

\section{Materials and methods}

Within this study, the cell-based Bionas 2500 Analyzing System [6] is used. The metabolic sensor chip of this system is able to detect the cellular changes in the morphology (cell impedance), the oxygen consumption rate (respiration) and the acidification rate (metabolic activity) of living mammalian cells in real-time. The human nasal cell line RPMI2650 was chosen for the experiments, as it is known from literature, that these cells are valuable model cell lines for nasal exposure [7]. The cells are grown on the surface of fibronectincoated metabolic sensor chips and form a confluent cell monolayer within 16-24 hours [4]. 
The fully covered chips are inserted in the biomodules of the analyzing system and connected to the reference electrode and the pumping system via a perfusion head. Liquid nutrient medium is pumped over the cells in an alternating $3 \mathrm{~min}$ stop/go cycle. Within the stop phase, the slope of the decrease of oxygen content in the medium as well as the decrease of the $\mathrm{pH}$ is detected and plotted as one data point per 6 minutes in the normalized respiration and acidification graphs. Difference in the slope of these curves yield information about the cellular status. For measurements of the effects of water-solved acetone, liquid acetone is diluted in Hepes-buffered DMEMbased running medium (RM). At the beginning, cells on the chip are supplied with untreated running medium via the pumping system to adapt the cells to the hydrodynamic shear stress of the liquid flow. After at least three hours adaption, the cells are exposed to acetone solutions.

For measurements of gaseous acetone, the nutrient medium above the cells is removed for a few minutes to enable a direct contact of the gas phase and the cell membrane. The gas is transported from a gas mixing station to the sensor chip. By means of a T-shaped gas exposure device, the gas stream flows over the cells and gets in contact with the cellular membrane via diffusion (see Fig. 1). Exposure durations of 10-15 minutes are sufficient to generate detectable changes in the cellular signals while the cells still stay alive. For all experiments, reference measurements have to be done to compare the effect of non-irritant humidified synthetic air $\left(\mathrm{O}_{2}: \mathrm{N}_{2} / 20: 80\right)$. Acetone gas is diluted in synthetic air to achieve the desired concentrations. The acetone gas samples are also humidified up to $60 \%$ relative humidity using a heated bubbler. After the gas exposure, liquid medium is re-added to the cells on the chip and the perfusion head is closed to reconnect the reference electrode and the pumping system.

\section{Results of liquid acetone measurement}

The response of RPMI 2650 towards liquid and gaseous acetone as a model substance for an organic compound was investigated. Acetone solved in water-based nutrient medium caused signal changes from the control measurements at concentrations of $2 \%$ (see Fig. 2). The impedance values are reduced in a concentration-dependant manner. This is in good accordance to the literature as acetone is highly lipophilic and therefore used in industry as a solvent. Changes in the respiration rate and in the acidification rate (data not shown) can be recovered instead of the impaired impedance, which hardly recovers after acetone treatment. The achieved results are in the range of standard cell culture cytotoxicity assays, like the MTT [8] assay and the LDH [9] assay. Both assay formats detect cytotoxic effects of acetone at concentrations of about $2 \%$ (data not shown).

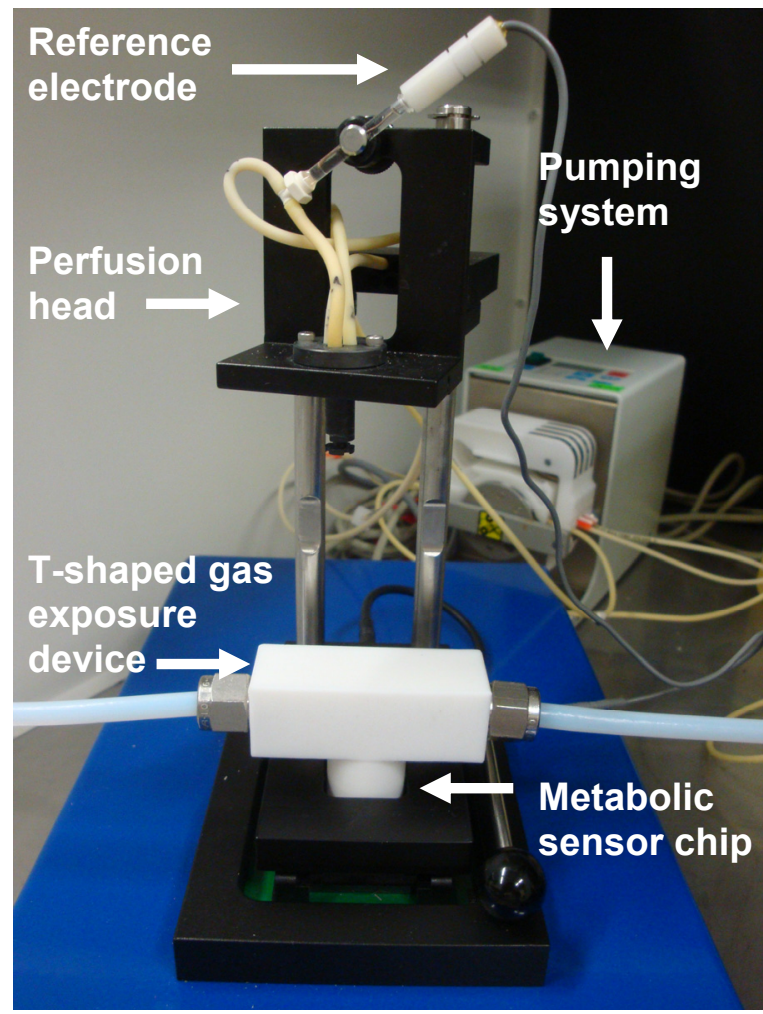

Fig. 1. Biomodule of the Bionas Analyzing System equipped with a T-shaped gas exposure device on a metabolic sensor chip. The perfusion head, which is connected to the nutrient medium delivering pumping system in the back and the reference electrode, is lifted during the direct gas exposure.

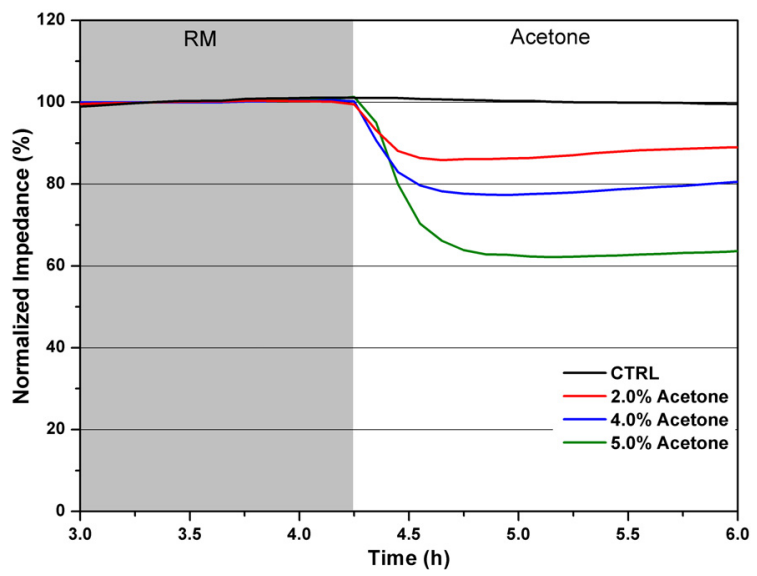

Fig. 2. Time transient of the impedance measurements of RPMI 2650 human nasal cells exposed to acetone solved in liquid culture medium after an adaption phase of $\sim 4$ hours with untreated culture medium (RM). The control (CTRL) measurement was constantly treated with running medium. 


\section{Results of gaseous acetone measurement}

Direct exposure of nasal cells to gaseous acetone causes changes in all three monitored cell parameters. The acetone concentrations that cause changes are in the range of several hundred ppm, being considerably lower than the concentrations needed to cause an effect when acetone is applied solved in nutrient medium. The direct exposure on the cellular membrane seems to have a much higher impact. Acetone is able to dissolve hydrophobic species like phospholipids out of the membrane. The results in Figure 3 show a concentration-dependent decrease of the impedance values after an exposure to gaseous acetone for 10 minutes, whereas synthetic air has no damaging effect on the cell layer integrity. The respiratory activity is increased in the presence of acetone gas (see Fig. 4). The immediate increase of the respiration after the end of the gas exposure recovers slightly over the following treatment with running medium, which indicates that no permanent change in the respiration system has been caused. An increase in the acidification rates of the nasal cells is observed too (see Fig. 5), although the increase in the cellular metabolism is smaller compared with the respiration.

The gas exposure parameters selected (humidity, flow rate, exposure time) are chosen to establish control measurements with synthetic air that hardly affects the cells. This simplifies the comparison with the test gases and ensures that no cell reaction occurs because of the exposure conditions. Repeated exposure towards synthetic air is possible without destruction of the cell monolayer on the chip surface (not shown). This is an essential precondition for the use of such a sensor system for the continuous monitoring of air samples.

\section{Conclusion}

The use of living mammalian cells grown on sensor electrodes for the detection of toxic gases is an emerging approach. Direct exposed cells react towards gaseous acetone in lower concentrations than towards solved liquid acetone. This sensor system might find application in the field of environmental monitoring of ambient air and aerosols as well as in the field of drug screening for respiratory drugs and narcotics.

\section{Acknowledgements}

The authors would like to emphasize special thanks to Christian Guijarro for his help with cell culture methods.

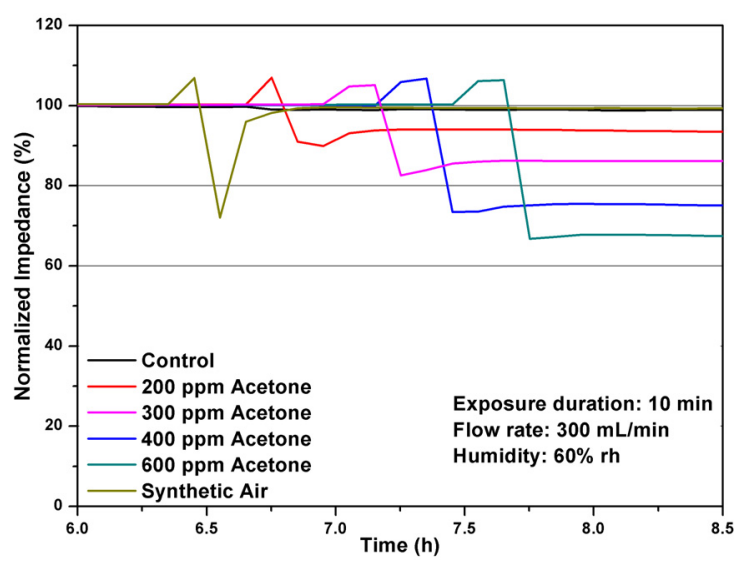

Fig. 3. Time transient of the impedance measurements of RPMI 2650 human nasal cells exposed to different concentrations of gaseous acetone in humidified synthetic air.

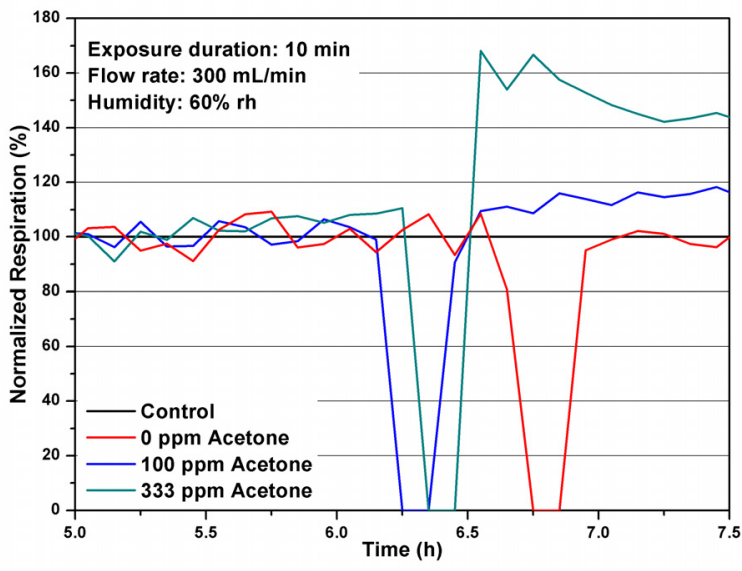

Fig. 4 Time transient of the oxygen consumption measurements of RPMI 2650 human nasal cells exposed to different concentrations of gaseous acetone in humidified synthetic air.

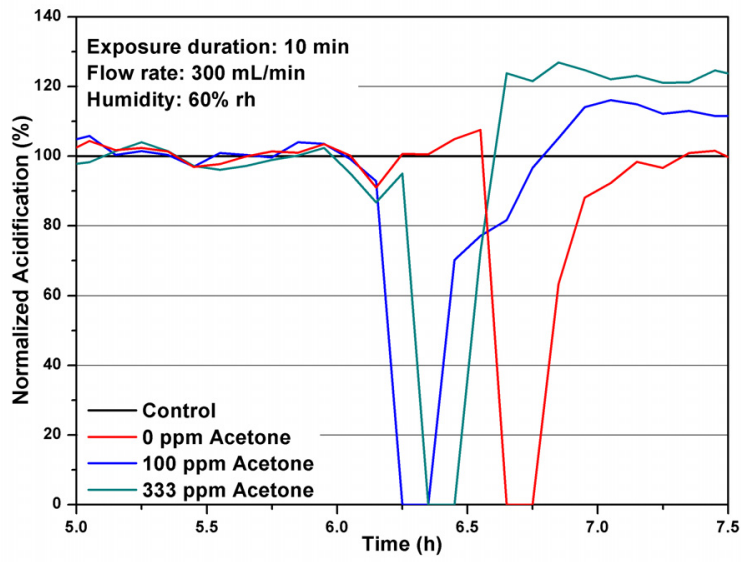

Fig. 5 Time transient of the acidification measurements of RPMI 2650 human nasal cells exposed to different concentrations of gaseous acetone in humidified synthetic air. 


\section{References}

[1] R. Kubisch, U. Bohrn, M. Fleischer, E. Stütz, Cellbased sensor system using L6 cells for broad band continuous pollutant monitoring in aquatic environments, Sensors 12, 3370-3393 (2012); doi:10.3390/s120303370

[2] H. Alborzinia, S. Can, P. Holenya, C. Scholl, E. Lederer, I. Kitonovic, S. Wölfl, Real-time monitoring of cisplatin-induced cell death, PLOS ONE 6, e19714 (2011); doi: 10.1371/journal.pone.0019714

[3] U. Bohrn, E. Stütz, M. Fleischer, M.J. Schöning, P. Wagner, Eukaryotic cell lines as a sensitive layer for rapid monitoring of carbon monoxide, Physica status solidi (a) 208, 1345-1350 (2011); doi: 10.1002/pssa.201000924

[4] U. Bohrn, E. Stütz, K. Fuchs, M. Fleischer, M.J. Schöning, P. Wagner, Air quality monitoring using a whole-cell based sensor system, Procedia Engineering 25, 1421-1424 (2011); doi: 10.1016/j.proeng.201112351

[5] J.H.E. Arts, J. Mojet, L.J. van Gemert, J.H.C.M. Lammers, J. Marquart, R.A. Woutersen, V.J. Feron, An analysis of human response to the irritancy of acetone vapors, Critical Reviews in Toxicology 32, 43-66 (2002); doi: $10.1080 / 20024091064174$

[6] L. Ceriotti, A. Kob, S. Drechsler, J. Ponti, E. Thedinga, P. Colpo, R. Ehret, F. Rossi, Online monitoring of BALB/3T3 metabolism and adhesion with multiparametric chip-based system, Analytical Biochemistry 371, 92-104 (2007); doi: 10.1016/j.ab.2007.07.014

[7] S. Bai, T. Yang, T.J. Abbruscato, F. Ahsan, Evaluation of human nasal RPMI 2650 cells grown at an air-liquid interface as a model for nasal drug transport studies, Journal of Pharmaceutical Sciences 97, 1165-1178 (2008); doi: 10.1002/jps.21031

[8] T. Mosmann, Rapid colorimetric assay for cellular growth and survival: application to proliferation and cytotoxicity assays. Journal of Immunological Methods 65, 55-63 (1983); doi: 10.1016/00221759(83)90303-4

[9] T. Sasaki, K. Kawai, K. Saijo-Kurita, T. Ohno, Detergent cytotoxicity: simplified assay of cytolysis by measuring LDH activity. Toxicology in Vitro 6, 451-457 (1992); doi: 10.1016/08872333(92)90052-S 